\title{
INVITED COMMENTARY ON Non-pharmacological interventions in dementia
}

In managing the behavioural and psychological symptoms of dementia (BPSD), clinical guidelines (Howard et al, 2001) and good clinical practice recommend that pharmacological interventions be used only after other, non-pharmacological, methods have been tried. In the real world, perhaps especially in care homes, neuroleptic medication is likely to be prescribed and continued, in many cases indefinitely. This necessarily selective review by Douglas and his colleagues perhaps illustrates some of the obstacles that must be addressed if the worthy intention to place less reliance on the illusory quickfix of the tranquilliser prescription is ever to become a reality (Douglas et al, 2004, this issue). The authors have identified a mixed bag of therapies with, at best, modest evidence for any efficacy, and which raise many issues regarding the feasibility of their widespread application.

\section{Need for clear treatment aims}

The various therapies described have a range of therapeutic goals, not always explicitly stated by those responsible for their development. Reality orientation, for example, has had a clear focus on cognition, particularly orientation. After falling from favour, largely through insensitive application, it has recently been adapted and revived as 'cognitive stimulation' (Woods, 2002). In a recent trial, the changes in cognitive function were of the same order of magnitude as those reported in trials of acetylcholinesterase inhibitors (Spector et al, 2003). In its new form, it is associated with improvements in quality of life, but there is no suggestion that it would, or should, affect BPSD. Reminiscence work has had a variety of aims, plausibly spanning both cognition (autobiographical memory) and mood. Validation therapy, with its emphasis on the emotional content of communication, similarly should have its main impact on affect. The 'alternative' therapies also have a range of potential aims, although the putative impact of aromatherapy and music therapy on arousal levels is of particular interest, if this is seen as a possible factor in behaviours described as agitated.

\section{Need for recognition of anxiety and depression as key challenges in dementia care}

The authors' comments on brief psychotherapies serve as a reminder of the importance of responding more actively to the well-documented high levels of anxiety and depression symptoms shown by people with dementia (Ballard et al, 1996a,b). It is an obvious step to apply well-established psychological therapies such as cognitive-behavioural therapy for depression (Scholey \& Woods, 2003) and relaxation for anxiety (Suhr et al, 1999) to people in the early stages of dementia who display such symptoms; there is now no doubt regarding the feasibility of such an approach, and one study successfully utilised family carers as therapists (Teri et al, 1997). More challenging are those people with severe dementia who have comorbid anxiety and depression; this is where some of the alternative therapies and activities may have most to contribute.

\section{BPSD v. challenging behaviour?}

The difference in terminology noted by Douglas et al reflects a fundamental difference in conceptualisation, which, if not addressed, could stifle the development of effective non-pharmacological approaches. In general, clinical psychologists prefer (although they are not completely happy with this) to talk of behaviour that challenges, because of its implicit reminder that the problem lies in part with our reaction to the behaviour, which of itself may not present a problem to the person with dementia. Challenging behaviour is a function of a particular care environment; in a different care setting, the behaviour in question may not be elicited, or may not be viewed as a problem by those providing care. The association between carer strain and difficult behaviour is often noted, but rarely is the possibility entertained that a stressed carer behaves in ways that elicit more difficult behaviour from the person with dementia (Woods, 2001). The implication is a difficult one; rather than prescribing a 'therapy', 
alternative or otherwise, some of our interventions have to involve changes in the attributions, attitudes and interactions of family carers and paid careworkers. It is here, indeed, that a fuller understanding of the carer's cognitions and affect regarding the person in their care, and also of their behaviour in relation to other members of the care system, will prove invaluable.

\section{Need for individualised formulations}

Having identified symptoms such as agitation, aggression, wandering and the like, it is tempting to identify the most effective therapeutic approach for each. However, the same label may become attached to quite different behaviours, requiring quite different interventions. Add to this the person's unique social environment, their particular profile of physical health, their life history and so on, and it is clear that an individual assessment and analysis of each person's situation is needed. Even if two people with dementia are both thought to be showing agitation because of a high level of internal arousal, what helps one may be quite different from what helps the other. One person's relaxing and calming hand-massage may be another person's invasion of personal space and confinement (Brooker et al, 1997). Evidence-based practice in dementia care entails establishing 'what works for whom', rather than a standard approach; this may require readiness to adopt a more empirical approach, using simple single-case designs, with the person as their own control.

\section{Implementing change}

If it is indeed the case that effective non-pharmacological interventions must be based on individual assessment and formulation, with an understanding of the role of the social environment in the development and maintenance of the problem, training alone, for family carers or for care-workers, will be of limited use. Input is also required from mental health professionals (including community mental health nurses and clinical psychologists, as well as psychiatrists) who have skills in dementia care and the ability to work at a systemic level. Some models of how this might be achieved are already being developed, for example through regular visits to a care home by a mental health nurse (Proctor et al, 1999; Ballard et al, 2002) or through the input of a multidisciplinary team in a family care context (Hinchliffe et al, 1995). Further analysis, development and evaluation of these intervention models is required, if non-pharmacological interventions are to take their proper place in the real world of dementia care.

\section{References}

Ballard, C. G., Bannister, C. \& Oyebode, F. (1996a) Depression in dementia sufferers. International Journal of Geriatric Psychiatry, 11, 507-515.

Ballard, C., Boyle, A., Bowler, C., et al (1996b) Anxiety disorders in dementia sufferers. International Journal of Geriatric Psychiatry, 11, 987-990.

Ballard, C., Powell, I., James, I., et al (2002) Can psychiatric liaison reduce neuroleptic use and reduce health service utilization for dementia patients residing in care facilities? International Journal of Geriatric Psychiatry, 17, 140-145.

Brooker, D. J. R., Snape, M., Johnson, E., et al (1997) Single case evaluation of the effects of aromatherapy and massage on disturbed behaviour in severe dementia. British Journal of Clinical Psychology, 36, 287-296.

Douglas, S., James, I. \& Ballard, C. (2004) Non-pharmacological interventions in dementia. Advances in Psychiatric Treatment, 10, 171-177.

Hinchliffe, A. C., Hyman, I. L., Blizard, B., et al (1995) Behavioural complications of dementia - can they be treated? International Journal of Geriatric Psychiatry, 10, 839847.

Howard, R., Ballard, C., O'Brien, J., et al (2001) Guidelines for the management of agitation in dementia. International Journal of Geriatric Psychiatry, 16, 714-717.

Proctor, R., Burns, A., Stratton-Powell, H., et al (1999) Behavioural management in nursing and residential homes: a randomised controlled trial. Lancet, 354, 26-29.

Scholey, K. A. \& Woods, B. T. (2003) A series of brief cognitive therapy interventions with people experiencing both dementia and depression: a description of techniques and common themes. Clinical Psychology and Psychotherapy, 10, 175-185.

Spector, A., Thorgrimsen, L., Woods, B., et al (2003) Efficacy of an evidence-based cognitive stimulation therapy programme for people with dementia: randomised controlled trial. British Journal of Psychiatry, 183, 248-254.

Suhr, J., Anderson, S. \& Tranel, D. (1999) Progressive muscle relaxation in the management of behavioural disturbance in Alzheimer's disease. Neuropsychological Rehabilitation, 9, 31-44.

Teri, L., Logsdon, R. G., Uomoto, J., et al (1997) Behavioral treatment of depression in dementia patients: a controlled clinical trial. Journals of Gerontology Series B - Psychological Sciences and Social Sciences, 52, P159-P166.

Woods, B. (2002) Editorial: Reality orientation: a welcome return? Age and Ageing, 31, 155-156.

Woods, R. T. (2001) Discovering the person with Alzheimer's disease: cognitive, emotional and behavioural aspects. Aging and Mental Health, 5 (suppl. 1), S7-S16.

Bob Woods Professor of Clinical Psychology with Older People, University of Wales Bangor (Ardudwy, Holyhead Road, Bangor, Gwynedd LL57 2PX, UK. E-mail: b.woods@ bangor.ac.uk), Co-Director of the Dementia Services Development Centre Wales and Director of the Centre for Social Policy Research and Development. 